
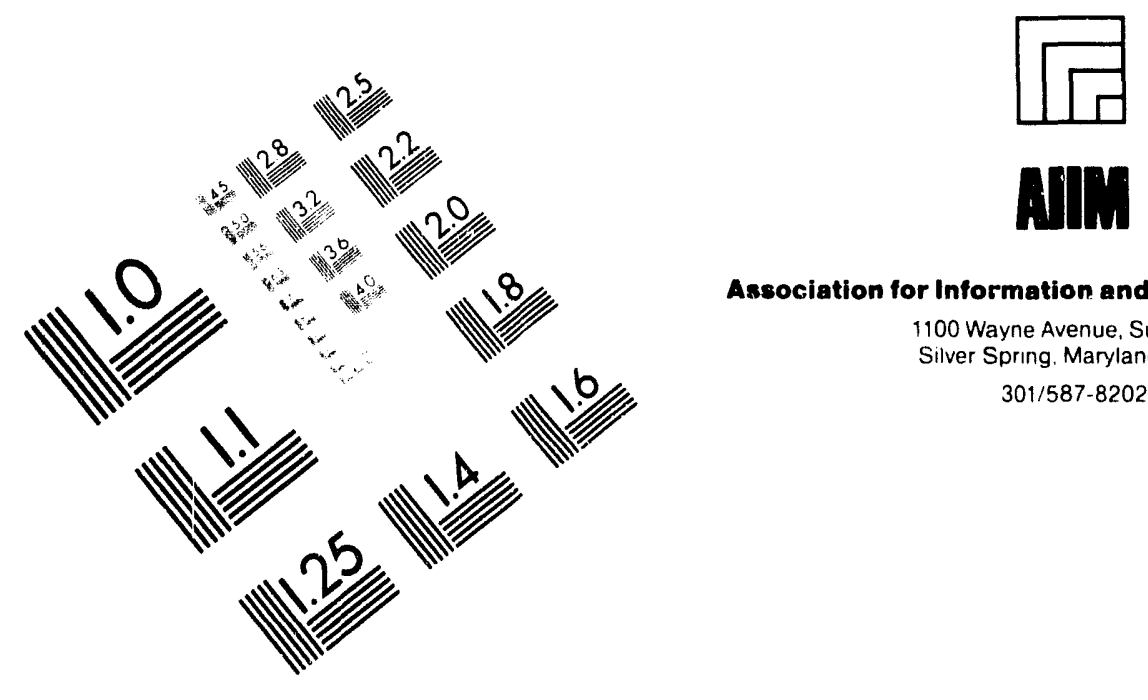

Association for Information and Image Management

1100 Wayne Avenue, Sulte 1100

Silver Spring. Maryland 20910

$$
\text { 301/587-8202 }
$$

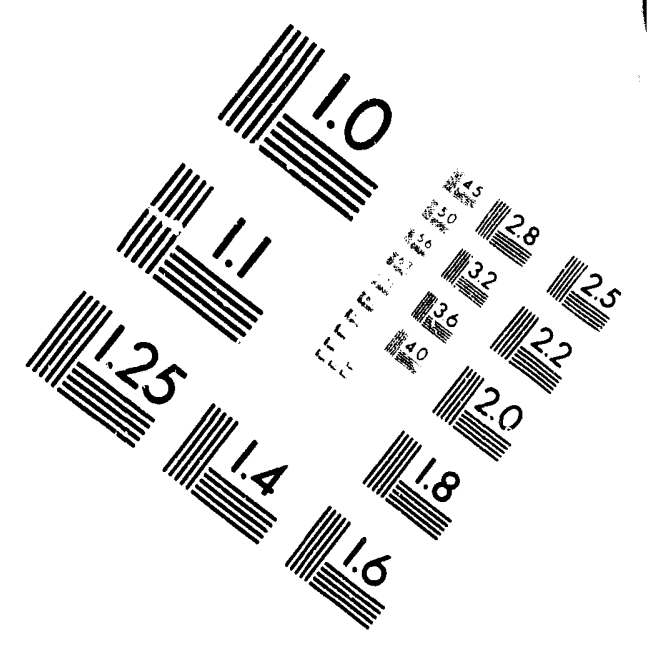

\title{
Centimeter
}

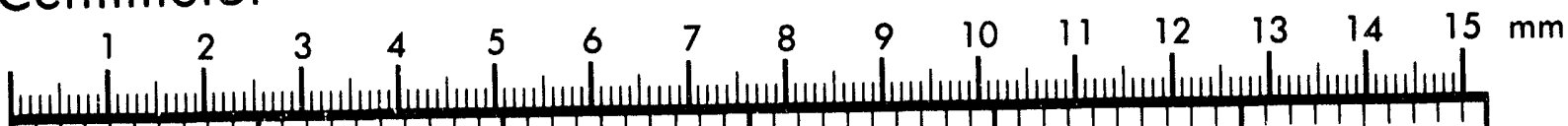

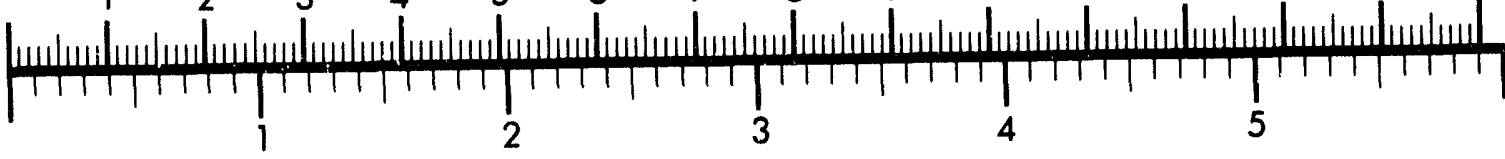
Inches
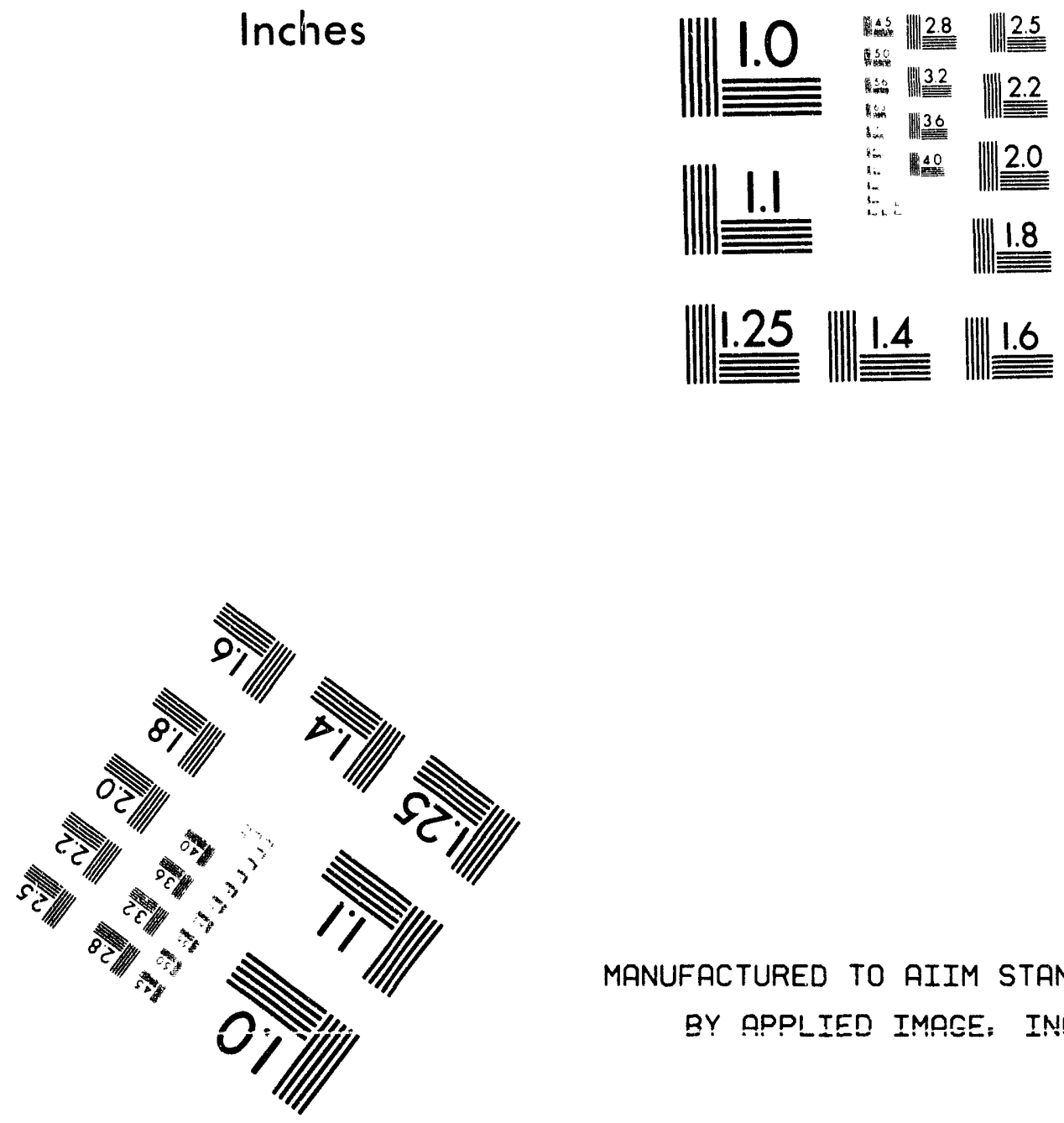

MANUFACTURED TO AIIM STANDARDS

BY PPPIIED IMPGE; INC.

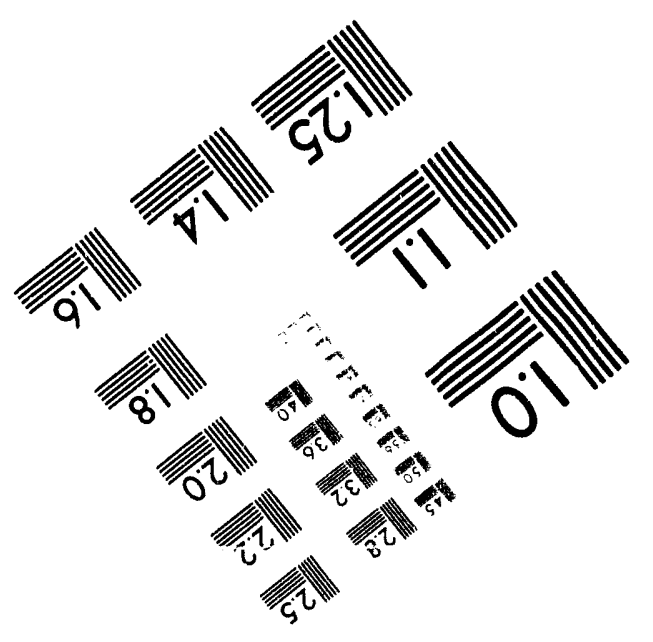



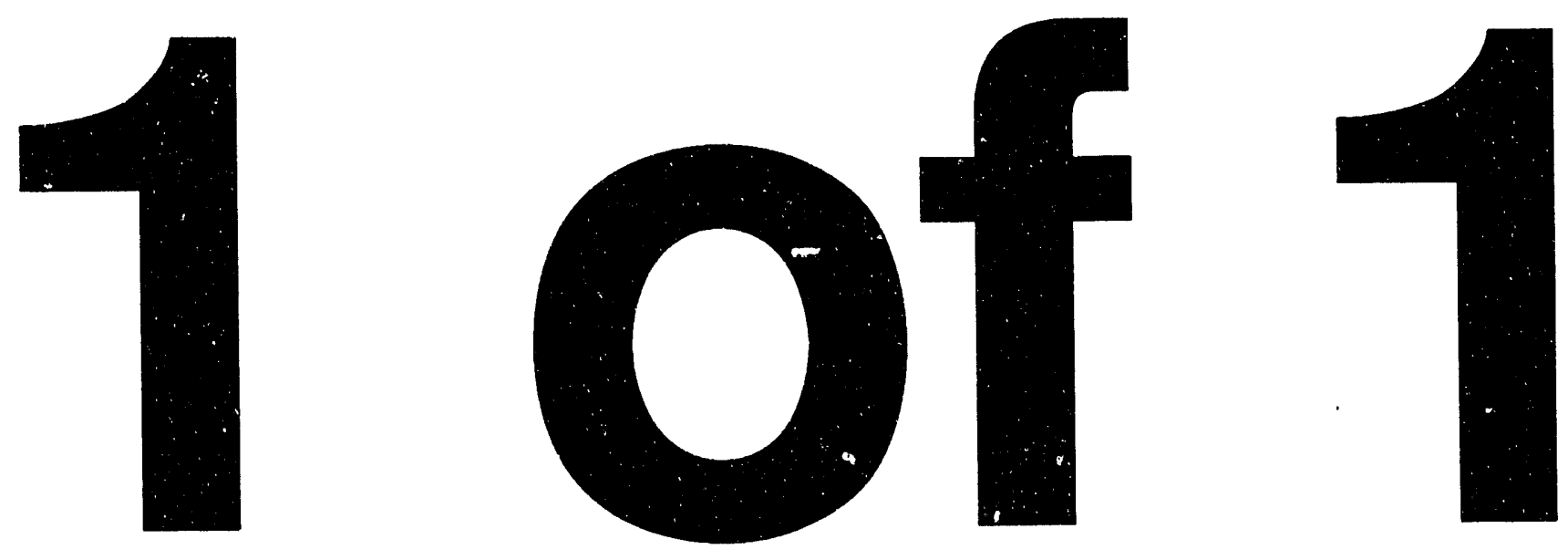


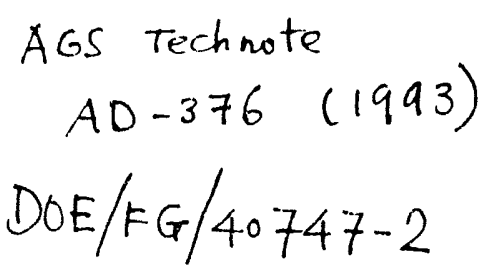

\section{Minimizing Linear Coupling in AGS with Skew Quads}

H. Huang, S.Y. Lee

Department of Physics, Indiana University, Bloomington, IN47405

L.G. Ratner

Brookhaven National Laboratory, Upton, NY11979

\section{Introduction}

Skew quadrupoles and solenoids can introduce linear betatron coupling into the beam. Linear coupling causes an energy exchange between the horizontal and vertical betatron motions. Such a process can lead to vertical emittance growth during the adiabatic betatron tune crossing. When two linear oscillators are coupled, one can find normal modes. The motion of these two normal modes are uncoupled. There exists separate Courant- Snyder invariants for these two normal modes. When the motion is projected onto $x-z$ planes, there is a smear. The betatron tunes of these two normal modes are separated by

$$
\Delta \nu=\sqrt{\left(\nu_{x}-\nu_{z}\right)^{2}+\left|C_{-}\right|^{2}}
$$

where

$C_{-}=\frac{1}{2 \pi} \int \sqrt{\beta_{x} \beta_{z}} e^{i\left[\mu_{z}-\mu_{z}-\left(\nu_{x}-\nu_{z}-x\right) \theta\right]}\left[\frac{a_{1}}{\rho}+\frac{M_{s}}{2}\left(\frac{\alpha_{x}}{\beta_{x}}-\frac{\alpha_{z}}{\beta_{z}}\right)-i \frac{M_{s}}{2}\left(\frac{1}{\beta_{x}}+\frac{1}{\beta_{z}}\right)\right] d s$

where

$$
a_{1} / \rho=\frac{\partial B_{z}}{\partial z} / B \rho, \quad M_{s}=B_{s} / B \rho
$$

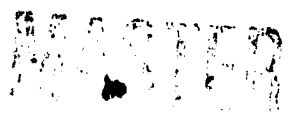


are skew quadrupole and solenoid strength, respectively, $p$ is an integer nearest to $\nu_{x}-\nu_{z}$.

The linear coupling causes emittance growth, so it should be minimized. Linear coupling caused by a solenoid can be corrected with skew quadrupoles by minimizing the coupling coefficient $C_{-}$. It requires two orthogonal skew quadrupole families.

The major source of linear coupling arises from the $5 \%$ partial solenoidal snake to be installed in the section I10 of the AGS. To correct the effect of linear coupling, we will use 4 families of skew quadrupoles located at (1) B17, D17, ...,L17; (2) A-L6; (3) A-L16; (4)A-L3. The A6 and A16 families are called weak skew quadrupoles.

\section{$C_{\text {_ }}$ of the Partial Snake}

For a snake, the coupling constant is

$$
\begin{aligned}
& C_{-}=\frac{M_{s}}{4 \pi} \int \sqrt{\beta_{x} \beta_{z}} e^{i\left[\mu_{x}-\mu_{z}-\left(\nu_{x}-\nu_{z}-p\right) \theta\right]}\left[\left(\frac{\alpha_{x}}{\beta_{x}}-\frac{\alpha_{z}}{\beta_{z}}\right)-i\left(\frac{1}{\beta_{x}}+\frac{1}{\beta_{z}}\right)\right] \theta_{s o l}(s) d s \\
& =\frac{M_{s}}{4 \pi} \int_{s_{0}}^{s_{0}+C} \sqrt{\beta_{x} \beta_{z}} e^{i\left[\mu_{x}-\mu_{x}-\left(\nu_{x}-\nu_{z}-p\right) \frac{\circ}{R}\right]}\left[\left(\frac{\alpha_{x}}{\beta_{x}}-\frac{\alpha_{z}}{\beta_{z}}\right)-i\left(\frac{1}{\beta_{x}}+\frac{1}{\beta_{z}}\right)\right] \theta_{s o l}(s) d s
\end{aligned}
$$

where the solenoid strength for the $5 \%$ snake is $M_{s}=0.05 \times 1.125 / 2.4384=$ $0.023 m^{-1}$, with $2.4384 m$ as the length of the solenoid, $s$ is the distance from the beginning point, and $\theta_{\text {sol }}(s)$ is a function defined as

$$
\begin{gathered}
\theta_{\text {sol }}(s)= \begin{cases}1 & \text { if s within snake } \\
0 & \text { otherwise. }\end{cases} \\
\nu_{x}=8.75, \nu_{z}=8.75, R=\frac{C}{2 \pi}=\frac{807.11}{2 \pi}=128.46 \mathrm{~m} \text {. Using the Simpson rule, }
\end{gathered}
$$

the integral from the snake is

$$
C_{-} \operatorname{lon}_{\text {on }}=-0.01216-i 0.00844=0.0148(-0.8216-i 0.5703)
$$


For $\nu_{x}=8.66667, \nu_{z}=8.66667$, the coupling coefficient is

$$
\left.C_{-}\right|_{\text {sn }}=-0.01075-i 0.0101=0.01475(-0.7288-i 0.6824)
$$

It shows that the coupling coefficient $\left.C_{-}\right|_{s n}$ is not sensitive to the betatron tune changes.

\section{$C_{-}$of the Skew Quadrupoles}

For skew quadrupoles, since a set of skew quadrupoles use a common power supply, they have the same strength. Using the thin lense approximation, the coupling constant is given by

$$
\begin{aligned}
C_{-} & =\left[\frac{a_{1}}{\rho}\right] \frac{1}{2 \pi} \int \sqrt{\beta_{x} \beta_{z}} e^{i\left[\mu_{z}-\mu_{z}-\left(\nu_{x}-\nu_{z}-p\right) \theta\right]} d s \\
& \doteq\left[\frac{a_{1}}{\rho} \Delta s\right] \frac{1}{2 \pi} \sum_{i=1}^{N} \sqrt{\beta_{x}\left(s_{i}\right) \beta_{z}\left(s_{i}\right)} e^{i\left[\mu_{x}\left(s_{i}\right)-\mu_{z}\left(s_{i}\right)-\left(\nu_{x}-\nu_{z}-p\right) \frac{s_{i}}{R}\right]}
\end{aligned}
$$

where $p$ is the integer part of $\nu_{x}-\nu_{z} . \mathrm{N}=6$ for skew quadrupoles in section $17, \mathrm{~N}=12$ for skew quadrupoles in section 3,6 and 16 . Then we get the $C_{-}$ for every skew quadrupoles family:

$$
\begin{aligned}
& \left.C_{-}\right|_{\text {sk3 }}=a_{3}(28.6566-i 2.9010)=a_{3} 28.8031(.9949-i .1007), \\
& \left.C_{-}\right|_{\text {ak6 }}=a_{6}(29.0680-i 6.1395)=a_{6} 29.7093(.9704-i .2067) \text {, } \\
& \left.C_{-}\right|_{\text {ak16 }}=a_{16}(29.9289+i 0.6021)=a_{16} 29.935(.9998+i .0201) \text {, } \\
& \left.C_{-}\right|_{\text {ek17 }}=a_{17}(14.5170-i 1.1866)=a_{17} 14.5654(.9967-i .0814)
\end{aligned}
$$

where $a_{i}=\left[\frac{a_{1}}{\rho} \Delta s\right]$ is the strength for every set of skew quadrupoles.

\section{Minimizing the Total $C_{-}$}

Minimizing the coupling requires at least two skew quadrupole families. First we consider the two 'strong' skew quadrupoles. It can be seen from the 
above equations that $\left.C_{-}\right|_{8 k 3}$ and $\left.C_{-}\right|_{8 k 17}$ are almost in phase. Since there are 20 sections in each superperiod, the skew quadrupoles in sections 3 and 17 are in symmetric positions, which gives rise to the in-phase effect. Due to this in-phase effect, we cannot just use the two 'strong' quadrupoles in sections 3 and 17 to compensate the coupling. From now on, we just consider the skew quadrupoles in sections 6,16 and 17.

Cancel the coupling means $\sum C_{-}=0$. Writing the real and imaginary parts individually, we get two linear equations:

$$
\begin{array}{r}
14.5170 a_{17}+29.9289 a_{16}+29.0680 a_{6}-0.01216=0 \\
-1.1866 a_{17}+0.6021 a_{16}-6.1395 a_{6}-0.00844=0
\end{array}
$$

The solutions of these linear equations are not unique. To use the skew quadrupoles most efficiently, the minimum values of skew quadrupoles strengths should be used. It is a linear-programming problem. Since there are only three parameters, we can use a simpler way to solve it.

Rewrite the first equation as

$$
a_{17}=\frac{1}{14.5170}\left(0.01216-29.9289 a_{16}-29.0680 a_{6}\right) m^{-1} \text {, }
$$

which is a plane in 3-D space as shown in Fig.1. Then substitute it into the second equation, we get

$$
a_{6}=0.2657\left(-0.00744+3.0485 a_{16}\right) m^{-1}
$$

which gives a straight line as shown in Fig.2. Minimum strengths corresponds to the point with smallest distance from the original point. It is $a_{16}=$ 


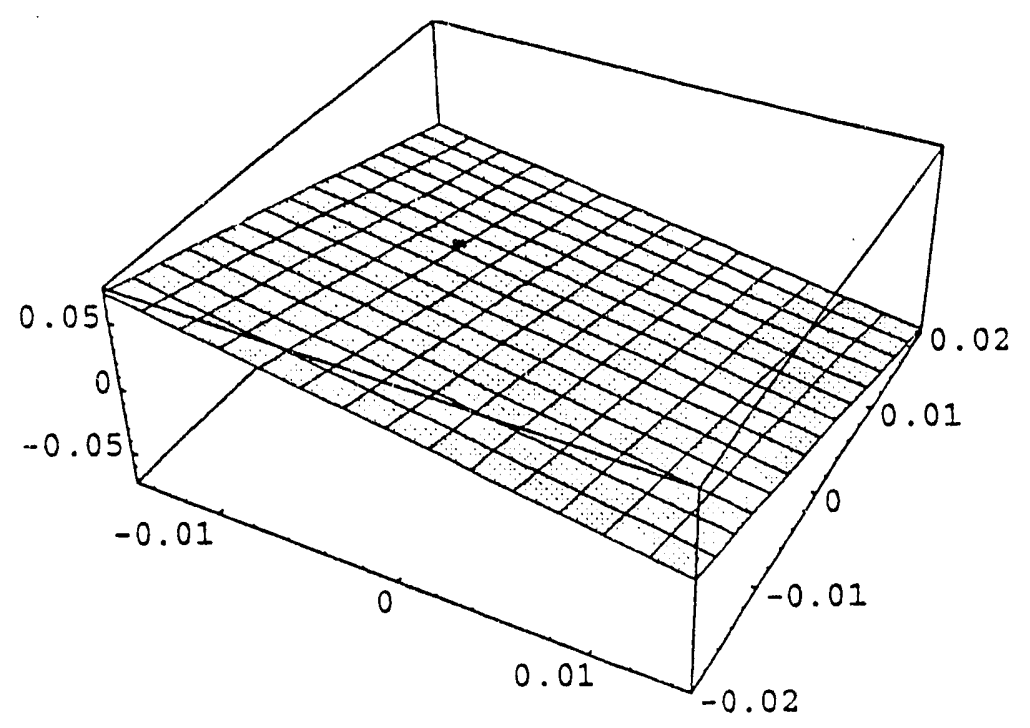

Figure 1:

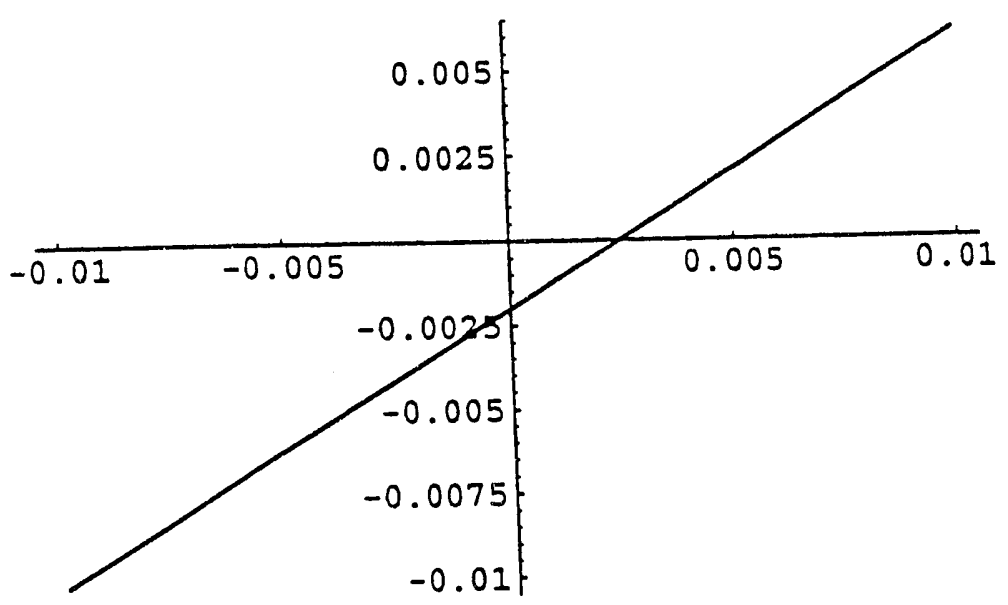

Figure 2: 
$0.00097 m^{-1}, a_{6}=-0.0012 m^{-1}$. Then $a_{17}$ can be calculated from the first equation: $a_{17}=0.00124 m^{-1}$.

Unfortunately, skew quadrupoles in sections 6 and 16 are 'weak' ones, the strength required cannot be achieved. The intrinsic resonance with highest energy corresponds to $G \gamma=36+\nu_{z}=44.75$, i.e., $p=23.4 G e V / c$ and $B \rho=78.0 T-m$. The maximum current of the skew quadrupoles in section 17 is $500 \mathrm{~A}$, corresponding $\int \frac{\partial B_{z}}{\partial z} d s=0.84 T$. So the maximum value of $a_{17}$ can not exceed

$$
\frac{0.84}{78.0}=0.0108 m^{-1}
$$

The value required for $a_{17}$ is smaller than this. The $\mathrm{B}$ fields of the skew quadrupoles in sections 6 and 16 are $0.0084 T$, so the maximum value of $a_{16}$ is

$$
\frac{0.0084}{78.0}=0.00011 m^{-1}
$$

Obviously, this is much smaller than the values required to cancel the coupling coefficient $C_{-}$of the snake. It means that the coupling can not be canceled by using the existing skew quadrupoles.

\section{Discussion}

To compensate the coupling caused by the $5 \%$ snake, new strong skew quadrupole families need to be installed. We need at least two families. To compensate the coupling efficiently, the skew quadrupole families should be located at positions with as large a phase difference as possible. Note that the coupling coefficient $\left.C_{-}\right|_{\text {sol }}$ from the solenoid has about equal magnitudes of real and imaginary parts, while the coupling coefficients from the quadrupole families are dominantly real except in the $2 \mathrm{nd}, 6 \mathrm{th}, 14$ th and 18 th straight 
sections. Since the coupling coefficients from these four straight sections are almost in-phase, we can choose only one of them, say the 6th. Another one should have as large a phase difference as possible from the 6th straight section. Actually, families 6 and 16 have the largest phase difference. So, the 'weak' skew quadrupoles in families 6 and 16 should be replaced by 'strong' skew quadrupoles. The strengthes can be calculated easily by using eqs.(1) and (2):

$$
a_{16}=0.00122 m^{-1}, \quad a_{6}=-0.00159 m^{-1}
$$

This means that the total skew quadrupole strength needed at the 6 th and the 16 th straight sections are

$$
12 a_{16}=0.0146 m^{-1}, \quad 12\left|a_{6}\right|=0.0191 m^{-1}
$$

i.e., we need only two strong skew quadrupoles to be located at the 6 th and at the 16 th straight sections to correct the linear coupling.

In addition to the coupling caused by the $5 \%$ snake, there also exists coupling caused by quadrupole misalignment. But the latter one is real part dominant and easier to be compensated for by the skew quadrupole families. The real coupling caused by both of them will be measured during the test run in August, 1993. After that, we will be able to determine the necessary coupling compensation scheme. 
Imaginary part of $\mathrm{C}_{-}$(Normalized)

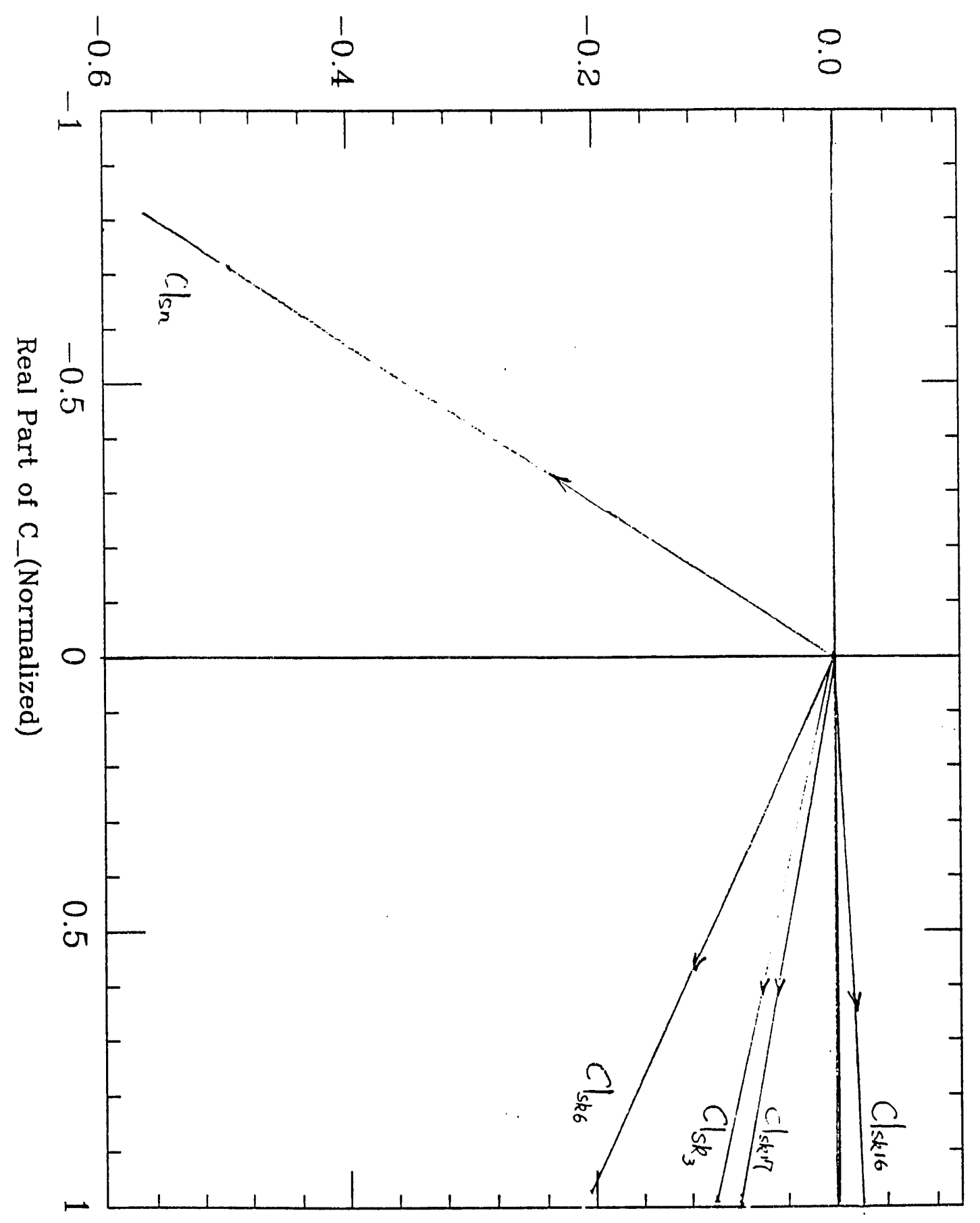



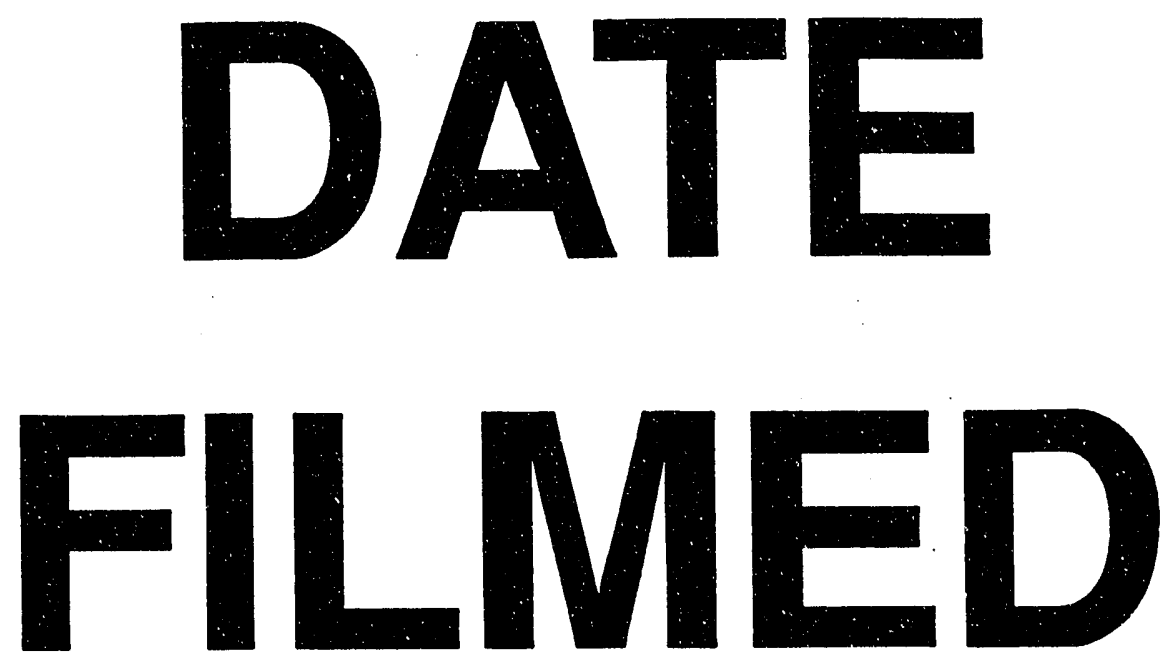

$8 / 17 / 93$
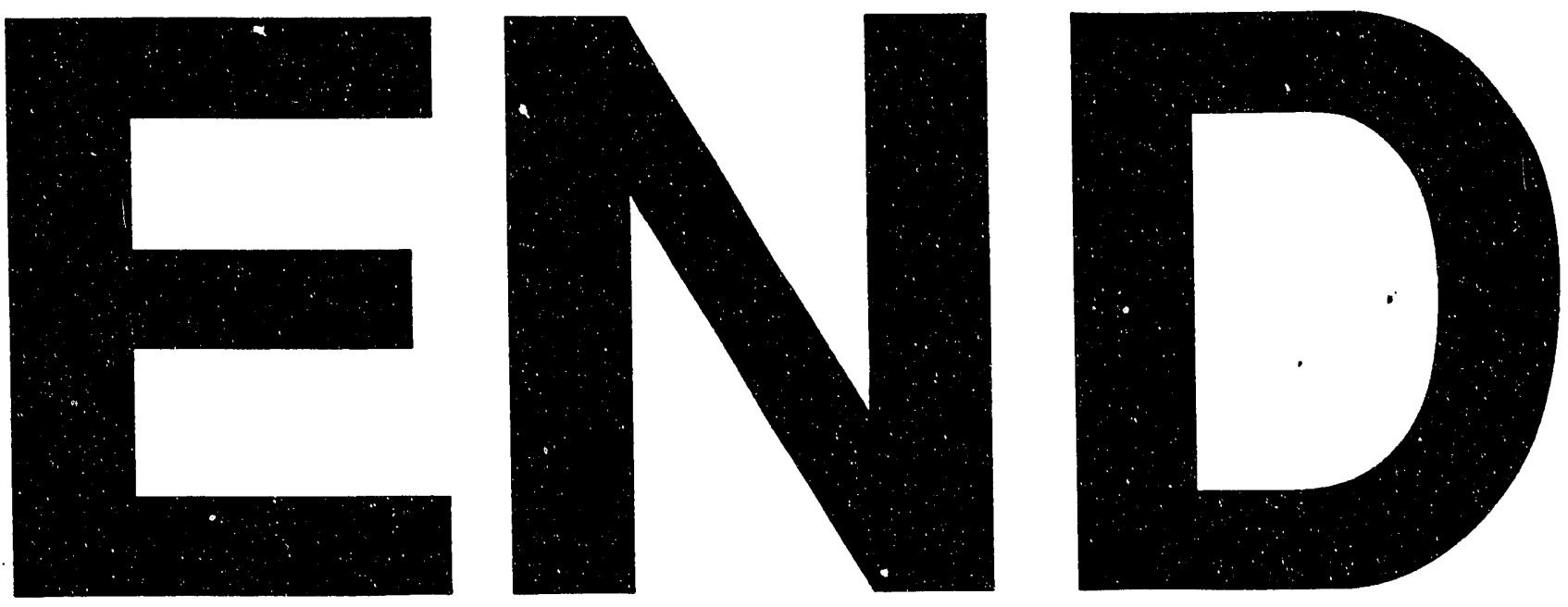
